Basrah Journal Of Surgery
Original Article

Bas J Surg, December, 26, 2020

\title{
GLOBAL COMPARISON OF THE OUTCOME OF SURGICAL TECHNIQUES FOR SPONDYLODISCITIS
}

\author{
Jessica Burton ${ }^{\circledR}$, Thamer Hamdan*, Bisola Ajayi", Mohsen Raza ${ }^{\$}$, Angelo \\ Fragkakis $^{\&}$, Cristina Lupu ${ }^{\prime}$, Timothy Bishop ${ }^{\%}$, Jason Bernard ${ }^{\prime} \&$ Darren Lui** .
}

${ }^{\circledR}$ MB,ChB student. *Professor of Orthopaedic Surgery at Basrah University, Visiting Professor at London Imperial College and Research Fellow as Saint George's Hospital London. "Orthopaedic and Spine Physician Associate. ${ }^{\$}$ Orthopaedic Registrar. ${ }^{\&}$ Spine Fellow. ^ Spine CNS. ${ }^{\circ}$ Orthopaedic Spinal Surgeon. 'Orthopaedic Spinal Surgeon. ${ }^{* *}$ Orthopaedic Spinal Surgeon. Department of Trauma and Orthopaedics, Saint George's University Hospital, London, UK.

\begin{abstract}
Spondylodiscitis is an infection of the intervertebral disc and neighbouring bone. It is treated conservatively or surgically using a range of techniques.

The aim of this review is to determine whether the surgical techniques between different countries have an effect on clinical outcome: postoperative complications, relapse rate, treatment failure rate or mortality rate.

Many articles were screened using Ovid and Pubmed databases for studies pertaining to the surgical treatment of spondylodiscitis. Paediatric studies, tubercular/brucellar/fungal/ postoperative infection and case reports were excluded from this review.

The results shows that no differences in the outcome of surgery between countries was found, and reasons for this along with solutions for moving forward with comparing surgical techniques worldwide are noted.
\end{abstract}

Key words: Spondylodiscitis, Surgery, Outcome, Comparison, Literature review.

\section{Introduction}

Q pondylodiscitis is a big umbrella term that covers spondylitis, discitis, and vertebral osteomyelitis. It is an infection of the vertebral body plus its neighbouring intervertebral disc. Haematogenous seeding is the most common route of infection but spread from adjacent infected sites and direct invasion are also possible ${ }^{1}$. Once Spondylodiscitis has been diagnosed, though often this is a prolonged process ${ }^{1}$, antibiotic therapy will begin and whether surgery or a more conservative approach is appropriate will be decided though there are no strict guidelines as to what treatment technique should be implemented such as antibiotic duration, which antibiotics, surgery type or immobilisation type ${ }^{2}$. This review only focuses on the difference between surgical techniques.

\section{Methods}

Literature Search
An electronic literature search of two online sources was done, Pubmed to find studies published within the last 10 years (January 2010 to January 2020) that are open access, and Ovid using 6 of their filters: Embase 1996 to 2020, Ovid MEDLINE ALL 1946 to January 31 2020, Global Health 1973 to 2020, PsycINFO 2002 to January 2020, Books@Ovid and Journals@Ovid Full Text. For both Pubmed and Ovid the search terms included; spondylodiscitis, vertebral osteomyelitis, discitis, or osteodiscitis and also included 'surgery' or 'treatment'. About 1116 articles were found, but after deleting all duplicates, only 434 articles were included.

Selecting Studies

All 434 articles' title and abstract were screened according to the exclusion criteria (figure 1). The titles and abstracts were analysed using inclusion and exclusion criteria. Included studies were those mentioning the surgical treatment 
of pyogenic spondylodiscitis in adult patients, prospective studies, case series and comparative studies. Any that mentioned tubercular, brucellar, fungal or postoperative spondylodiscitis were excluded along with case reports, reviews, meta analyses especially if they were non-specific about causative agents, and also paediatric patients were excluded. Three hundred ninety five studies were excluded based on this criteria and 39 studies were included for full text assessment. A further 28 studies were excluded after reading the full articles as assessing the full text revealed one or more patients in each study with tubercular spondylodiscits, fungal spondylodiscitis, postoperative infection or the study was more of literature review. This left us with 11 for analysis of data on conservative and surgical treatment. See table I for studies selected for analysis and table II for their clinical data.

Figure 1: Articles screening method

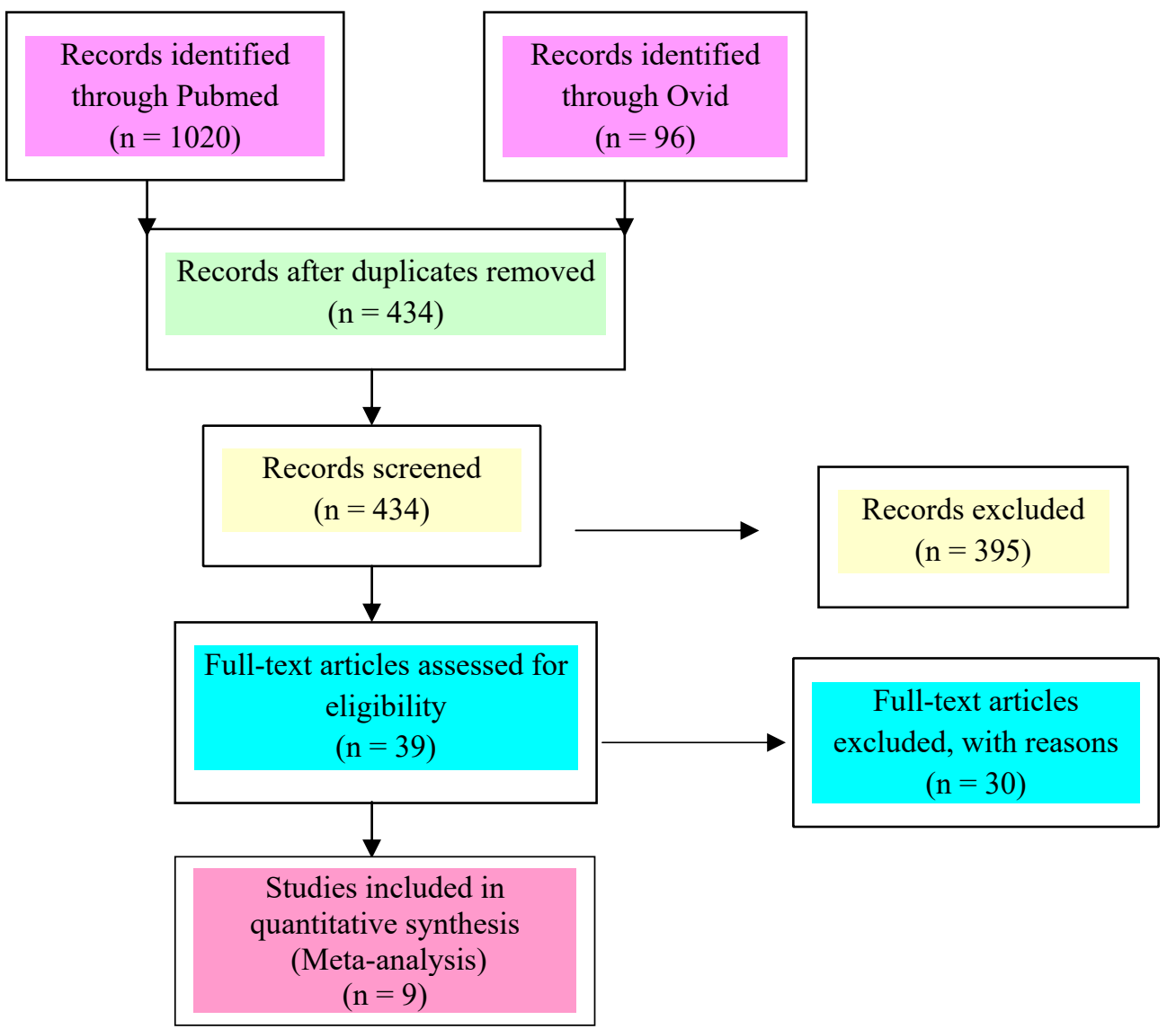

Table I ${ }^{3-11}:$ Studies selected for analysis

\begin{tabular}{lllllll}
\hline Author & $\begin{array}{l}\text { Publication } \\
\text { year }\end{array}$ & $\begin{array}{l}\text { Country } \\
\text { studied }\end{array}$ & Study design & $\begin{array}{l}\text { Number of } \\
\text { patients }\end{array}$ & $\begin{array}{l}\text { Mean } \\
\text { age }\end{array}$ & $\begin{array}{l}\text { Sex } \\
\text { (M:F) }\end{array}$ \\
\hline Anagnostakos & 2013 & Germany & Retrospective & 8 & 68.8 & $3: 5$ \\
Arnold & 2014 & USA & Retrospective & 94 & 55 & $66: 28$ \\
Griffith-Jones & 2018 & UK & Retrospective & 10 & 67 & $7: 3$ \\
Hempelmann & 2010 & Germany & Retrospective & 18 & 72 & $7: 11$ \\
Lee & 2016 & Taiwan & Retrospective & 54 & 60 & $42: 12$ \\
Li & 2017 & China & Retrospective & 14 & 57.4 & $9: 5$ \\
Lin & 2014 & Taiwan & Retrospective & 45 & 62.2 & $25: 20$ \\
Tsai & 2017 & Taiwan & Retrospective & 43 & 58.9 & $28: 15$ \\
Valancius & 2012 & Denmark & Retrospective & 117 & 61 & $66: 51$ \\
\hline
\end{tabular}


Table II $^{3-11}$ : Clinical data of selected studies

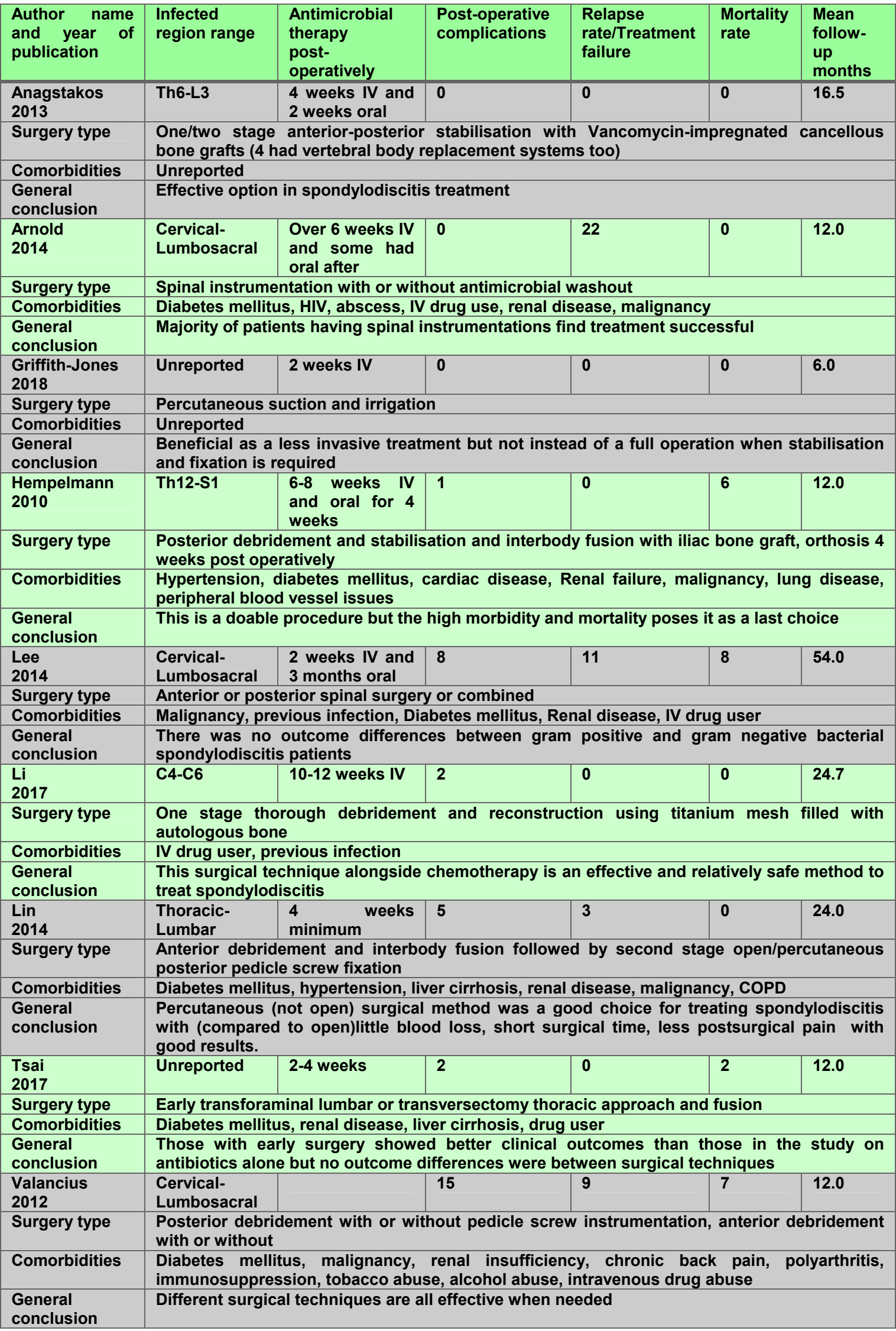




\section{Results}

\section{China}

A total of 14 patients were studied in China that fit our criteria using 1 stage debridement with a titanium mesh cage filled with implanted autologous bone graft. Whilst there was no treatment failure, relapses or fatalities there were $2 / 14(14.2 \%)$ patients with postoperative complications ${ }^{3}$. This technique used in China was effective for eradicating the infection.

\section{Denmark}

A total of 117 patients were studied in Denmark that fit criteria. Four different surgical techniques were used: posterior debridement with or without pedicle screw fixation and anterior debridement with or without pedicle screw fixation. In this larger study in their surgical group, there were $22 / 117 \quad(18.8 \%) \quad$ with postoperative complications, 3 who had treatment failure or a relapse of the infection $(2.6 \%)$ and $7 / 117(6.0 \%)$ ultimately died either in hospital or during their follow up period with various causes ${ }^{4}$. All these surgical techniques are suitable for treating spondylodiscitis with such low treatment failure, relapse and mortality rates.

\section{Germany}

A total of 26 patients were studied in Germany that fit criteria. They were treated using one of 2 techniques. The first technique being a 1 or 2 stage anteriorposterior stabilisation with Vancomycinimpregnated cancellous bone grafts, with or without a vertebral body replacement system $^{5}$. The second technique being posterior stabilisation and interbody fusion using an iliac bone graft ${ }^{6}$. Unfortunately, postoperative complications were not stated in 1 study so could be assumed as no patients but the other reported only 1 patient, so it was $1 / 26(5.6 \%)^{6}$ postoperative complication rate overall. Relapse or treatment failure was none ${ }^{5,6}$. The mortality rate was 6 in one study so overall $6 / 26$
$(23 \%)^{6}$. Ultimately Germany saw a relatively high mortality rate with the surgical techniques studied yet low postoperative complication, relapse and treatment failure rates ${ }^{5,6}$. Usually a higher mortality rate would suggest the surgery is inappropriate when there are already techniques available that have less fatalities but one potential reason for this high mortality rate could be because they were all in the same study which was looking at very vulnerable patients in age and comorbidities. This is more suggestive that those particular patients were at risk so skews the results for Germany somewhat.

\section{Taiwan}

Three studies suited the criteria providing a total of 142 patients that were studied in Taiwan. They were treated using several surgical techniques: anterior or posterior surgery or combined ${ }^{7}$, anterior debridement and interbody fusion followed by second stage open or percutaneous posterior pedicle screw fixation ${ }^{8}$, early transforaminal lumbar or transversectomy thoracic approach and fusion, anterior radical debridement and fusion and finally anterior procedure with posterior instrumentation and fusion'. A $15 / 142(10.6 \%)$ patients suffered with postoperative complications, 14/142 (9.9\%) relapsed or the treatment failed and 10/142 $(7.0 \%)$ patients died after their surgery ${ }^{7-9}$. Overall, all of these techniques were effective at eliminating infection with relatively low mortality rates too.

\section{$U K$}

A total of 10 patients were studied in the UK that met the criteria. They were treated using percutaneous suction and irrigation. The study reported zero postoperative complications, no relapses or treatment failure and a $0 \%$ mortality rate ${ }^{10}$.

\section{USA}

A total of 94 patients were studied in the USA that met the criteria. They were treated 
using spinal instrumentation with or without antimicrobial irrigation. Postoperatively no complications were present in any patient, 22/94 (23.4\%) experienced relapse or treatment failure and no patients died ${ }^{11}$. This high relapse or failure rate has unclear reasons especially alongside the lack of patients with postoperative complications and no deaths.

\section{Discussion}

Lannelongue is thought to be the first doctor to describe spondylodiscitis in $1879^{12}$ but symptoms that have been credited to infection of the spine were noted as early as $1200 \mathrm{BC}^{13}$.

Many factors may increase a person's risk and susceptibility to developing spondylodiscitis which can be split into patient factors and surgical factors. Patient factors include but are not limited to; increasing age, liver cirrhosis, kidney failure, immunosuppression, diabetes mellitus and rheumatic diseases. There are also patient lifestyle factors that include alcoholism and IV drug use but also lifestyle factors that may contribute to the development of the above conditions ${ }^{14}$. Surgical risk factors include previous spinal surgery and indwelling intravascular lines ${ }^{14}$.

Epidemiologically, spondylodiscitis affects 4 to 24 people per $1,000,000$ people in developed countries ${ }^{15}$ with an interesting bimodal age spread with peaks at under 20 years of age and again at around 50-70 years of age ${ }^{13}$. Spondylodiscitis affects many more males with a male to female ratio ranging from $2: 1$ to $5: 1.13$

Typically spondylodiscitis is caused by singular bacteria. The most common causative bacterial agent is Staphylococcus aureus closely followed by gram-negative bacteria including Escheria coli. S. aureus typically infects by intrusion cutaneously through wounds ${ }^{16}$. Interestingly almost $1 / 3$ of discitis cases are post-operative or following a spinal procedure with no links to specific techniques ${ }^{13}$. These are the causative agents resulting in pyogenic spondylodiscitis. Other possible aetiologies are granulomatous (encompassing tuberculous, brucellar, fungal) and parasitic although these will not be focused on in this article.

The three main routes for spondylodiscitis development are; haematogenous seeding, spread from adjacent infected sites and direct invasion postoperatively ${ }^{11}$.

The most common route is the haematogenous seeding via the vertebral arterial supply ${ }^{12}$. In adults because their intervertebral discs are avascular and their intraosseous arteries have terminal points, a septic embolus results in infarcted tissue. The resulting infection spread causes spondylodiscitis $^{12}$. Posterior spinal elements are rarely affected by this route due to their poor blood supply so when they are involved it is more likely to be other types of spondylodiscitis or an alternative route of the pyogenic type ${ }^{12}$. Once the vertebrae and/or disc are infected it can spread to surrounding tissues, the epidural space and nerve roots resulting in bone structural integrity weakening and even being destroyed, abscess formation and other tissue destruction ${ }^{17}$.

Unfortunately, spondylodiscitis remains difficult to detect due to it's generic clinical presentation, a fact recognised in many articles. Gradual in its onset, it presents as back pain primarily ${ }^{18}$ that is worst at night and exacerbated by exercise, percussion locally to infected area, straightening their spine from being at an incline and knee flexion $^{19}$. But since so many of the population have low back pain (84 in 100 people experience this at some point in their life and this doesn't even include other regions of the back ${ }^{20}$ and 1 in 15 people see their primary care doctor about that back pain, it proves difficult to distinguish the early presentation of spondylodiscitis from the rest of people experiencing less severe causes of their back pain. Additionally, fever might occur although only up to $50 \%$ of 
patients $^{12}$, along with several neurological symptoms: sensory loss, weakness and radiculopathy one of which is seen in $1 / 3$ of patients ${ }^{17}$. A phenomenon commonly seen is due to spondylodiscitis often having a haematogenous seeding route (when not a post-operative presentation) many signs and symptoms present may be due to the initial infection such as urinary tract infection, skin or soft tissue infection ${ }^{14}$.

Such a wide range of unspecific symptoms, not even limited to those mentioned above, leads to an often significant delay in correct diagnosis, as much as up to 6 months ${ }^{1}$. Laboratory investigations with an extremely high sensitivity for infection are erythrocyte sedimentation rate (ESR) and C-reactive protein (CRP). Both are found above their reference ranges in over $9 / 10$ cases of spondylodiscitis. But despite these findings, they are not sufficient to identify spinal infection in particular due to lacking specificity as a diagnostic test ${ }^{12}$. A test that is amongst the normal haematological screen for infection is the white blood cell count but this isn't a beneficial diagnostic marker in spondylodiscitis due to it being within normal range in over $60 \%$ of cases ${ }^{17}$, so ESR and CRP are considered more useful. Ascertaining which pathogen is responsible is one of the most beneficial and specific testing due aiding treatment choices and may identify how the infection started. Blood culturing is the first port of call since pyogenic spondylodiscitis commonly has only one causative agent and a haematogenous route. It contains a useful result in $40-70 \%$ of cases ${ }^{1}$ but the range of positive results could be as wide $25-89 \%$ depending on the article.

When blood culturing doesn't yield a useful result, biopsy is undertaken next ${ }^{18}$. There are few different types of biopsy but the primarily used techniques in spondylodiscitis are CT-guided biopsy (percutaneous) and spinal biopsy

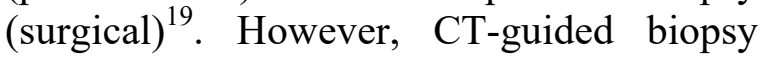
could be even less successful at identifying the microorganism than blood cultures or may be more helpful as the percentage of positive results ranges from $19 \%$ to $100 \%$ depending on the study ${ }^{12}$. What has been shown though as a more consistent microbiological diagnostic test is the increase in positive results when open or surgical techniques are used to obtain the biopsy as the pathogen detection success rate is as high as $68-93 \%{ }^{19}$.

Radiological imaging may also be beneficial to use in the diagnosis of spondylodiscitis as it can rule out differentials that are detected, usually by Magnetic Resonance Imaging (MRI) ${ }^{17}$ since this has high specificity and accuracy, both over $90 \%{ }^{18}$. But even when spondylodiscitis is suspected imaging can still be helpful. Even though the infection itself is not detectable the structural effects of spondylodiscitis such as inflammation signs like oedema, destruction of the vertebral end-plates, abscess presence ${ }^{14}$, and collapse of the disc spaces ${ }^{19}$ may be picked up that point toward Spondylodiscitis as the final diagnosis.

Treatment of pyogenic spondylodiscitis can broadly be divided into conservative antimicrobial treatments and surgical intervention ${ }^{17}$. The aims of this method are to eliminate the causative agents of infection, maintain or increases spinal stability and pain reduction ${ }^{12}$. Therapy begins once the pathogen has been successfully identified so an appropriate antibiotic can be selected but it has not been decided what length of antibiotic course is the most effective ${ }^{19}$ although duration of antibiotic treatment is extended for patients at risk ${ }^{2}$.

There are no standard guidelines for treating spondylodiscitis since there is such variation depending on the patients' needs ${ }^{2}$ but typically all patients will need antibiotic therapy even if they then go on to have surgery which makes antibiotics a cornerstone in treating spondylodiscitis. However, the antibiotics used need to be 
carefully considered due to the intervertebral disc being avascular from the age of 16-21 so antibiotics need to diffuse from a nearby blood vessel. One factor that affects this is charge. The intervertebral disc contains negatively charged groups which creates an electrical gradient so when this is combined with the lack of antibiotics naturally occurring in the intervertebral disc creating a concentration gradient, positively charged antibiotics are more likely to penetrate the disc than negatively charged antibiotics ${ }^{20,21}$. Alongside the antibiotics, usually where surgical stabilisation is not considered, conservative treatment of analgesia and bed rest or immobilisation is used to take pressure of the affected vertebrae ${ }^{19}$.

Those who are put forward for surgery are patients who aren't responding to conservative treatment, those with neurological deficits that are progressing, compromised vertebral stability, abscess presence or extensive bony structure damage due to the infection ${ }^{14}$. The aim of surgery can be split into two focuses; the removal of infected, damaged or even necrotic tissue, and then the implanting of instruments or fusing bones to support the spine postsurgery to allow normal function. There are several different surgical techniques available to achieve the removal of tissue for spondylodiscitis such as laminectomy, decompression and debridement, all of which can be approached anteriorly or posteriorly. It depends on the patient presentation as to which is used. Other elements that may take place as well include fusion, use of synthesised materials or the patients' own tissue and instrumentation which are all also done anteriorly or posteriorly as well. This whole process may require multiple operations to each side of the spine but sometimes it is just a single surgery ${ }^{12,14,22}$.

It is the comparison of success of different surgical technique across countries that will be focussed on in this review. Anterior decompression and debridement is a common surgery for spondylodiscitis to excise the infected and damaged tissue. This will be followed by a bone graft, cage insertion and/or fusion to provide the stabilisation a spine needs ${ }^{22}$. The stabilisation is often provided by instruments so to reduce the chances of postoperative infection titanium is used and the debridement step is accompanied by an 'antibiotic barrier' ${ }^{2}$. A posterior approach is used in certain situations. For example if multiple segments are involved, posterior stabilisation is recommended to prevent postoperative complications including the graft migrating or deformity developing ${ }^{22}$. Other uses for posterior approach include lumbar epidural abscess which might also require fusion or instrumentation for stabilisation and non-complicated thoracolumbar infections ${ }^{22}$.

The overall mortality rate for pyogenic spondylodiscitis varies between papers but may range from $0-29 \%^{12,14}$. Mortality may be due to age and its co-morbidities ${ }^{14}$ or early on in the disease progression sepsis is likely to be the cause ${ }^{12}$. Complications due to spondylodiscitis may include lasting neurological deficits (in up to $33 \%$ of patients), uncontrollable pain (up to $33 \%$ of patients) or relapse of the condition ${ }^{12}$.

\section{Evaluation}

Strengths of this review include countries such as Taiwan and the USA having sufficiently large cohorts, 142 and 94 respectively. The larger study sample which means more accurate values for the outcome are likely and outliers are less likely to skew the data as much. However, the actual number of studies per country that we reviewed is poor with Taiwan only having 3 , Germany having only 2 and the other 4 countries having only 1 each. This suggests the surgical techniques and outcomes may not be representative of each country overall and more studies that fit the inclusion and exclusion criteria should be found to provide more accurate data for each country. Another problem with this review is 
although the PRISMA guidelines were mostly followed in the deduplication, screening and full text assessment, there was no quality assessment performed on the final studies chosen nor did we check their respective references. Studies could have been used that didn't have enough evidence, poor ethics or variables that were not controlled or accounted for. Spondylodiscitis represents between 3\% and $5 \%$ of all cases of osteomyelitis ${ }^{20}$, it is a rare condition so not many studies are published with large sample sizes that also meet all our criteria so it is difficult to find enough appropriate studies for a review of this type. Spondylodiscitis is a condition with a lot of risk factors that can provide postoperative complications such as diabetes mellitus that extends healing times and increases vulnerability to infection so this could be one factor increasing relapse rates. There are many risk factors that may also skew the outcome of patients, so looking at surgical technique alone may mask these confounding variables.
In conclusion, there is little to no difference in surgical outcome when treating spondylodiscitis across different countries. No surgical technique stood out as more effective with less postoperative problems in any of the studies using the three measurements suggesting that any technique may be appropriate. Therefore, it may be beneficial to study more in depth the other clinical outcome in different countries to further analyse their surgical techniques such as length of hospital stay, neurological deficits and quality of life opposed to only postoperative complication, relapse, treatment failure and mortality rates. This may reveal a technique or more that is the most effective and safe. Alternatively, it could be more suitable to compare the surgical techniques themselves or compare the specific centres providing the treatment as it is apparent that surgical techniques varies greatly within the countries so viewing this as a whole could be limiting findings in this review.

\section{Reference}

1. Homagk L, Marmelstein D, Homagk N, Hofmann GO. SponDT (Spondylodiscitis Diagnosis and Treatment): spondylodiscitis scoring system. J Orthop Surg Res. 2019 Apr; Available from: https://www.ncbi.nlm.nih.gov/pmc/articles/PMC6458762/

2. Sobottke R, Seifert H, Fätkenheuer G, Schmidt M, Goßmann A, Eysel P. Current Diagnosis and Treatment of Spondylodiscitis. Dtsch Arztebl Int [Internet]. 2008 Mar;105(10):181-7.

3. Li H, Chen Z, Yong Z, Li X, Huang Y, Wu D. Emergency 1-stage anterior approach for cervical spine infection complicated by epidural abscess. Medicine (Baltimore) 2017 Jun 30;96(26).

4. Valancius K, Hansen ES, Høy K, Helmig P, Niedermann B, Bünger C. Failure modes in conservative and surgical management of infectious spondylodiscitis. Eur Spine J. 2013 Aug;22(8):1837-44.

5. Anagnostakos K, Koch K. Pharmacokinetic Properties and Systemic Safety of VancomycinImpregnated Cancellous Bone Grafts in the Treatment of Spondylodiscitis. Biomed Res Int. 2013 [cited $2020 \mathrm{Jul}$ 8]. Available from: https://www.ncbi.nlm.nih.gov/pmc/articles/PMC3730214/

6. Hempelmann RG, Mater E, Schön R. Septic hematogenous lumbar spondylodiscitis in elderly patients with multiple risk factors: efficacy of posterior stabilization and interbody fusion with iliac crest bone graft. Eur Spine J. 2010 Oct;19(10):1720-7.

7. Lee C-Y, Wu M-H, Cheng C-C, Huang T-J, Huang T-Y, Lee C-Y, Huang J-C, Li Y-Y. Comparison of gram-negative and gram-positive hematogenous pyogenic spondylodiscitis: clinical characteristics and outcomes of treatment. BMC Infect Dis. 2016. Available from: https://www.ncbi.nlm.nih.gov/pmc/articles/PMC5139091. 
8. Lin T-Y, Tsai T-T, Lu M-L, Niu C-C, Hsieh M-K, Fu T-S, Lai P-L, Chen LL-H, Chen W-J. Comparison of two-stage open versus percutaneous pedicle screw fixation in treating pyogenic spondylodiscitis. BMC Musculoskelet Disord. 2014 Dec 18. Available from: https://www.ncbi.nlm.nih.gov/pmc/articles/PMC4300775/

9. Tsai T-T, Yang S-C, Niu C-C, Lai P-L, Lee M-H, Chen L-H, Chen W-J. Early surgery with antibiotics treatment had better clinical outcomes than antibiotics treatment alone in patients with pyogenic spondylodiscitis: a retrospective cohort study. BMC Musculoskelet Disord. 2017 Apr $27 ; 18$. Available from: https://www.ncbi.nlm.nih.gov/pmc/articles/PMC5408454/

10. Griffith-Jones W, Nasto LA, Pola E, Stokes OM, Mehdian H. Percutaneous suction and irrigation for the treatment of recalcitrant pyogenic spondylodiscitis. J Orthop Traumatol. 2018 Dec ;19(1).

11. Arnold R, Rock C, Croft L, Gilliam BL, Morgan DJ. Factors Associated with Treatment Failure in Vertebral Osteomyelitis Requiring Spinal Instrumentation. Antimicrob Agents Chemother. 2014 Feb;58(2):880-4.

12. Gouliouris T, Aliyu SH, Brown NM. Spondylodiscitis: update on diagnosis and management. J Antimicrob Chemother. 2010 Nov 1 : 65(suppl_3):iii11-24.

13. Hamdan TA, Chasib RJ, Mssadi AK. Spinal infection chapt.4. Ahmad Kadem Al-Ali Press, Basrah Iraq. 2016.

14. Nickerson EK, Sinha R. Vertebral osteomyelitis in adults: an update. Br Med Bull. 2016 Mar 1;117(1):121-38.

15. Gentile L, Benazzo F, De Rosa F, Boriani S, Dallagiacoma G, Franceschetti G, Gaeta M, Cuzzocrea F. A systematic review: characteristics, complications and treatment of spondylodiscitis. European Review. 2019. Available from: https://www.europeanreview.org/article/17481

16. Liu GY. Molecular Pathogenesis of Staphylococcus aureus Infection. Pediatr Res. 2009;65:71-77.

17. Graeber A, Cecava ND. Vertebral Osteomyelitis. In: StatPearls. Treasure Island (FL): StatPearls Publishing; 2020. Available from: http://www.ncbi.nlm.nih.gov/books/NBK532256/

18. Marchionni E, Marconi L, Ruinato D, Zamparini E, Gasbarrini A, Viale P. Spondylodiscitis: is really all well defined? European Review. 2019. Available from: https://www.europeanreview.org/article/17494

19. Herren C, Jung N, Pishnamaz M, Breuninger M, Siewe J, Sobottke R. Spondylodiscitis: Diagnosis and Treatment Options. Dtsch Arztebl Int. 2017 Dec;114(51-52):875-82.

20. Care (UK) NCC for P. Low Back Pain. Royal College of General Practitioners (UK); 2009. Available from: https://www.ncbi.nlm.nih.gov/books/NBK11702/

21. Zhu Q, Gao X, Li N, Gu W, Eismont F, Brown MD. Kinetics of Charged Antibiotic Penetration into Human Intervertebral Discs: A Numerical Study. J Biomech. 2016 Sep 6;49(13):3079-84.

22. Mavrogenis AF, Megaloikonomos PD, Igoumenou VG, Panagopoulos GN, Giannitsioti E, Papadopoulos A, Papagelopoulos PJ. Spondylodiscitis revisited. EFORT Open Rev. 2017 Nov;2(11):447-61. 